\title{
In-situ colour correction for digital images acquired under non-standard lighting conditions
}

\author{
P. M. K. Alahakoon ${ }^{2}$ and Annamalai Manickavasagan ${ }^{1 *}$
}

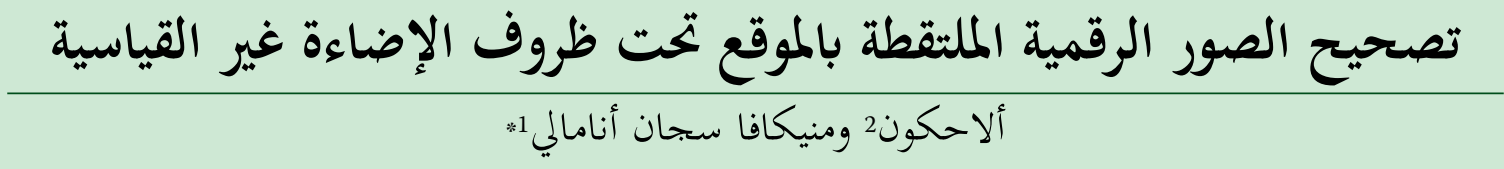

Abstract. Computer vision techniques using colour images are becoming popular in food and agriculture sector. Need of a standard illumination source is an important criterion in this approach to determine various attributes based on RGB values of the objects. In general, under laboratory conditions with standard lighting, an imaging system performs with high consistency in digitizing colour. However, in field conditions where the availability of a standard light source cannot be guaranteed, the colour interpretations may not yield accurate results. The objective of this study was to develop a simple algorithm to compensate for the variations in RGB values due to varying light conditions. It is intended to be useful in situations where taking digital images of objects without standard light sources is essential for a particular purpose. A set of quadratic transformation algorithms were developed to transform the RGB values of the images acquired under five different lighting conditions. The mean variance in RGB values of the image of a colour palette (with 6 different colours) taken under five lighting conditions were in the range of $277-548$. After implementing the developed algorithm, this was reduced to $34-142$. Similarly, this variance was reduced from $180-294$ to $63-128$ in the test conducted with a plant material. This algorithm can be easily adopted in all computer vision applications where variations in colour interpretations due to nonstandard lighting sources are common.

KEYwORDS: Colour balancing; illumination; image correction; computer vision.

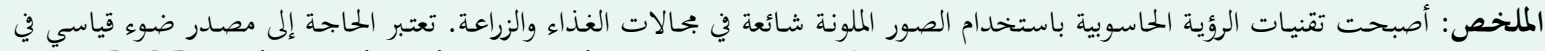

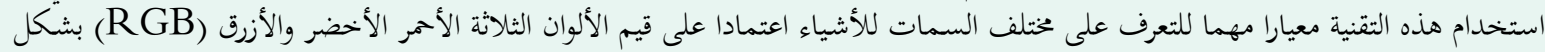

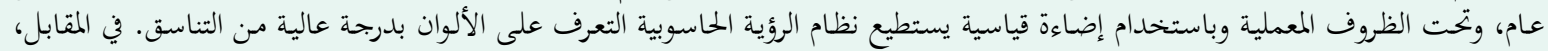

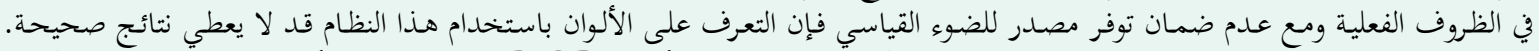

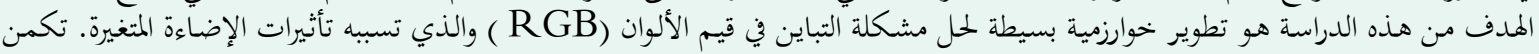

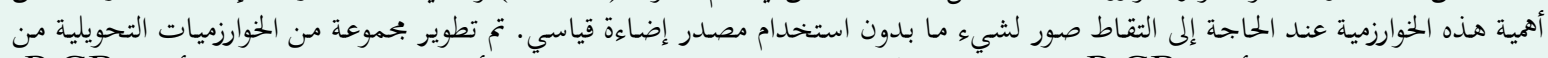

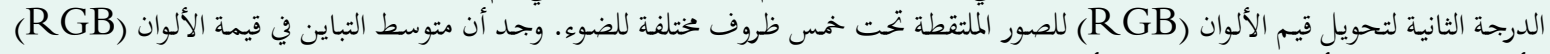

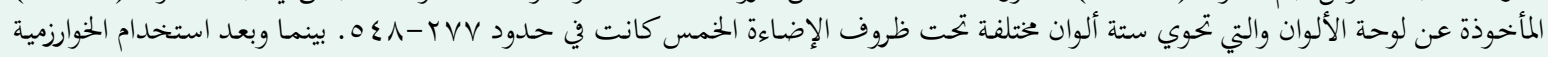

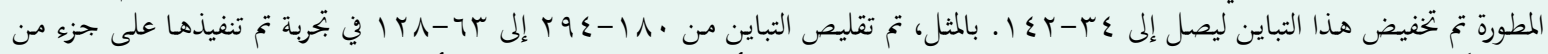

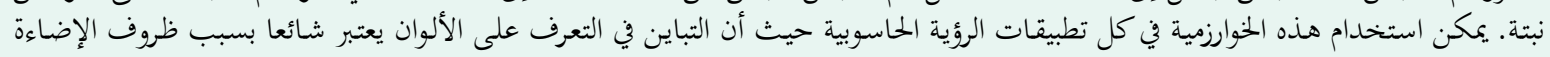
غير القياسية والمتغيرة. الكلمات المفتاحية: موازنة اللون؛ الإضاءة؛ تصحيح الصورة؛ الرؤية الحاسوبية

\section{Introduction}

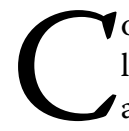

omputer vision techniques have been successfully used in various pre-harvest and post-harvest applications of agricultural and food products. Due to the common availability of digital imaging equipment and image processing software, many have been encouraged to develop their own techniques which are more inexpensive and easy to use (O'Neal et al., 2002) than depending on commercially available equipment.

\footnotetext{
$1_{*}$ A.Manickavasagan (1) Department of Soils, Water and Agricultural Engineering, College of Agricultural and Marine Sciences Sultan Qaboos University, P.Box 34, PC 123, Sultanate of Oman. ${ }^{2}$ Department of Agricultural Engineering, Faculty of Agriculture, University of Peradeniya, Sri Lanka.
}

Further, the developments in digital imagery have opened many new trends in plant and food applications. Digital cameras in combination with computers and appropriate software can be used to image and evaluate the objects for their many surface qualities with relative ease and at an affordable cost. However, the researchers are compelled to acquire images under laboratory conditions, by illuminating the test sample under standard lighting conditions in order to achieve a consistent colour interpretation which is a must for the success of subsequent analyses.

The selection of the illumination source must be done carefully which may drastically change the outcome (Luo et al., 1997; Brown and Timm, 1992; Manickavasagan et al., 2008). Due to this reason, most of the image related studies inevitably use either a laboratory 
setup to acquire images under controlled lighting conditions or use a commercially available flat bed scanner to scan the objects for obtaining their images in the case of flat objects such as plant leaves (Murakami et al., 2005). Some of the light sources used are circular fluorescent lights (Paliwal et al., 2003; Majumdar and Jayas,1999), fluorescent tube lights (Manickavasagan et al., 2008), incandescent lights, and infrared lights (halogen lamps) in special situations (Hehn and Sokhansanj, 1990; Liu et al. 2005). Digital imaging is very much dependent on the light source and the resulting interpretation of colours in RGB coordinates or as gray levels.

However, in agriculture like many other natural science fields, there are many instances where the sample or the object needs to be imaged in the natural environment. In field conditions, the plant materials such as leaves, fruits and flowers have to be imaged without removing from the plant. Also in food handling and in supply chain applications, there are several situations where the background lighting cannot be made standard all the time.

One of the challenges faced by researchers in taking digital images under such non-standard lighting situations that may also change from one picture to another, is to maintain closeness in the representative colours in the images. Even though there are white balancing options included into many modern digital cameras used today in order to provide a close approximation to the actual colours present in the scene, capabilities in the camera alone are not sufficient to yield the same 'digital' colours for an object imaged under different lighting situations. Even though professional software packages provide excellent improvement in colour and contrast, the resulting colours may not yield the same RGB values for the same object imaged under different environments and lighting situations.

On the other hand, since the colour of the sample or its specific regions of interest are represented as an RGB value, the researcher must be able to ensure that the acquired digital image does contain RGB values very close to the actual if not exact. The expected actual RGB values are the ones that will be obtained for a lighting situation that will provide 0, 0, 0 for black and 255, 255, 255 for white regions in the same scene. Therefore, it is essential that the digital images acquired are properly preprocessed in such a way that RGB values are closer to the actual as that will assist in proper diagnosis and drawing conclusions on the image properties, especially in food and agriculture related work which involves field images. As can be seen in some applications, measurement of different colour patches is important in making decisions in a large number of studies (O'Neal et al., 2002).

Shahin and Symons (2003) developed an algorithm to adjust the colours recorded by digital scanners in order to establish uniform interpretation of colours in the event of using more than one scanner for scanning grains for research purposes. Chang and Reid (1996) presented a method for correcting for the variations in RGB values caused by vision system components used in acquiring the image. Different types of error sources have been identified and modeled in order to correct for the errors. Weng et al. (2006) proposed a new algorithm for automatic white balancing of digital images which provided more visually appealing high contrast images compared to some of the existing methods such as gray world method, perfecto reflector method, fuzzy rule method, and Chikane's method. Conversely, Chikane and Fuh (2006) also presented an improved algorithm that can be incorporated into the image processing software in digital cameras, showing that white balancing is a prime requirement of all image acquisition operations.

In order to carry out manual white balancing, a more artistic operation, it is necessary to acquire pictures in the raw format, and use professional software customized to post-adjust the RGB components of the image based on the information recorded by the camera at the time of acquisition. In situations where raw image acquisition and the use of professional graphics processing software is limited, especially in industrial visits, and acquisition of images in agricultural fields, this approach posed limitations in use, thus justifying an attempt to develop techniques to address this issue.

Takemura and Ishii (2011) presented a neural network based approach for determination of actual colour of objects for robot vision systems. Due to its very sophisticated and demanding computational power requirement, normal users find it difficult to adopt such to their work. Besides, the calibrations performed are camera specific and may not be applicable for different cameras used under different conditions. Li et al. (2006) developed a specific colour adjustment system to obtain close to true colour representations of colposcopic images used in auto diagnosis of cervical cancer and also in telemedicine related applications. Since the diagnosis is primarily based on the colour of the tissue observed under artificial light sources, they have found that variations in the light source provided different colours in the digital image acquired, which may obstruct reaching a clear and fast diagnosis. Development of a simple algorithm that can be applied for each image would be highly beneficial to utilize in computer vision techniques for food and agricultural applications where huge variations in lighting conditions are inevitable. Therefore the objective of this study was to develop an algorithm to correct the RGB values of an image based on the RGB values of three standard colour cards imaged under the same lighting condition. 


\section{Methodology}

\section{Standard colour cards}

Black, gray and white standard colour cards (DGK Colour Tools, Boston, Massachusetts, USA) were used as the basis for correcting the RGB values of different regions of the images acquired under different lighting situations. The homogeneous RGB values of these colour cards were $(0,0,0),(128,128,128)$ and $(255,255,255)$ for black, white and gray, respectively.

\section{Colour palette}

A flat colour palette comprising of 6 different colour regions along with the 3 standard colour cards was assembled by using uniform colour sheets as shown in (Fig. 1). The colours were selected by taking 3 stronger shades (blue, green and red) and three lighter shades (yellow, light green and pink) to test the effectiveness of the algorithm.

\section{Image acquisition}

A digital colour camera (Resolution: $4608 \times 3456$; Model: WB850F; Samsung Electronics Company Ltd., UK) was used to acquire images. The developed colour palette was imaged under five non-standard lighting conditions in order to simulate real field conditions: (1) shade of a building; (2) fluorescent tube lighting (inside the laboratory; (3) natural cloudy (outside); (4) in a dark corridor (imaged with built-in flash); and (5) focused fluorescent lighting (test bench)

\section{Field test}

To investigate the performance of the developed algorithm, a simulated field experiment was conducted by taking images of a plant leaf under the same lighting situations. In this experiment, the plant leaf was imaged along with the three standard colour cards in all lighting conditions.

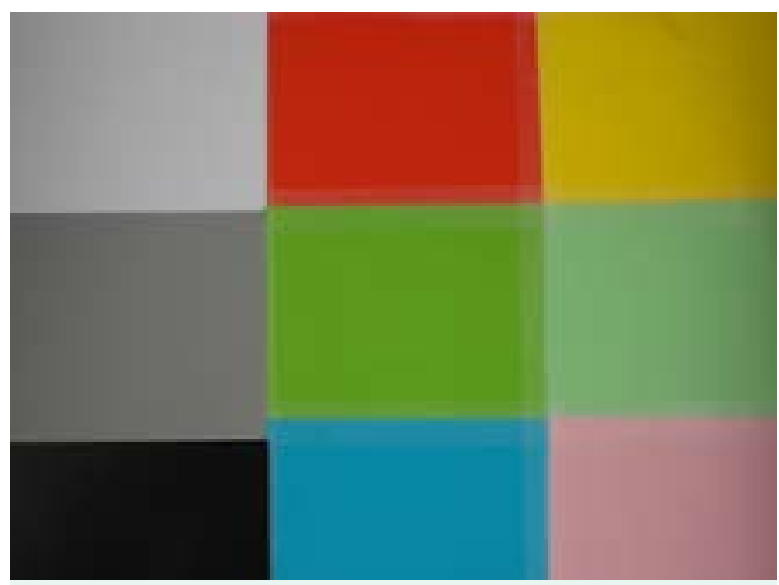

Figure 1. Image of the developed colour palette (first column represents 3 standard colour cards).

\section{Results and discussion}

\section{Algorithm development with standard colour cards}

The colour palette, comprising of 6 different colour squares and 3 standard colour cards were used together and exposed to 5 lighting situations. RGB values extracted from the standard colour cards were used to develop a quadratic transformation algorithm for each image using the least squares method, so that the RGB values for the three cards would be translated to $(0,0,0),(255,255$, $255)$, and $(128,128,128)$, respectively for Black, White, and Gray, as prescribed by the manufacturers. All the mathematical operations related to algorithm development and numerical computations were done in MS Excel environment.

The algorithm development is briefly described by considering the matrices $[\mathrm{Y}],[\mathrm{X}]$ and $[\mathrm{h}]$, where $[\mathrm{Y}]$ containing the expected gray levels for the standard colours [0 128 255], [X] containing the actual measured RGB values representing each colour, placed as [R2 R1] for the equation for the $\mathrm{R}$ component, and [h] matrix being the coefficients required as the solution for each equa-

Table 1. Variance observed in the RGB values among the images acquired under 5 different lighting conditions before and after correction.

\begin{tabular}{|lllllll|} 
& \multicolumn{2}{c}{ Red } & \multicolumn{2}{c}{ Green } & \multicolumn{2}{c|}{ Blue } \\
\hline Card colour & Before & After & Before & After & Before & After \\
\hline Red & 817 & 0 & 80 & 285 & 61 & 101 \\
Yellow & 868 & 0 & 595 & 11 & 0 & 0 \\
Green & 398 & 117 & 390 & 78 & 122 & 605 \\
Light green & 336 & 42 & 525 & 12 & 366 & 27 \\
Blue & 68 & 42 & 504 & 218 & 511 & 64 \\
Pink & 798 & 3 & 600 & 111 & 604 & 57 \\
Mean & 548 & 34 & 449 & 119 & 277 & 142 \\
\hline
\end{tabular}




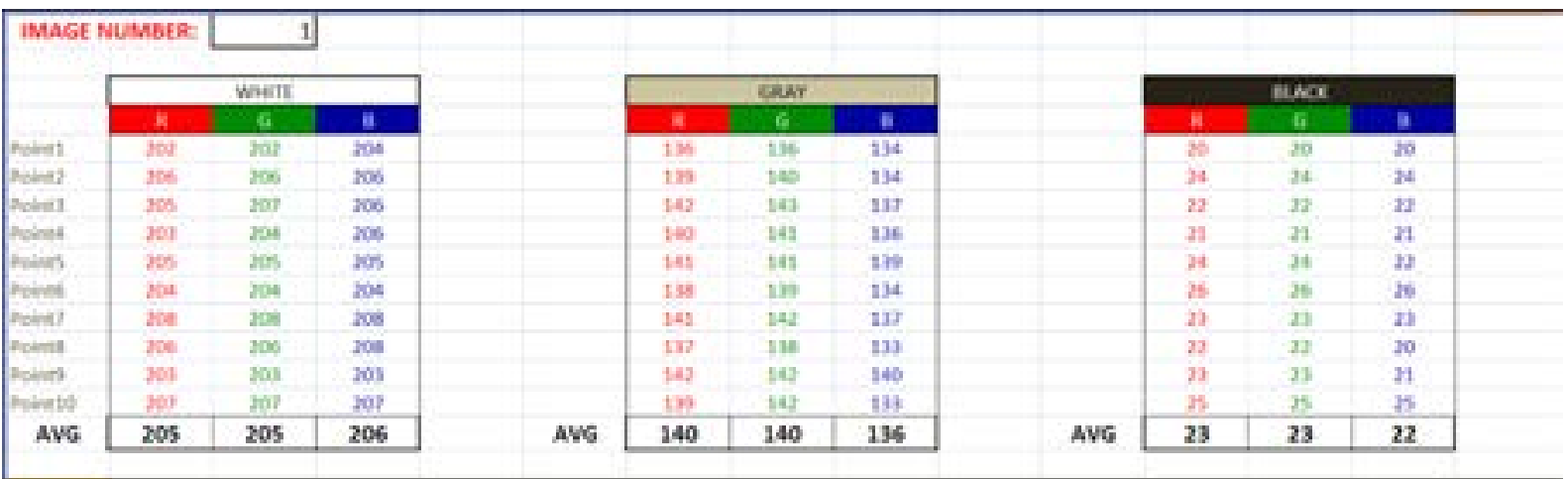

Figure 2. Example of the RGB values representing three standard colour cards (White, Gray, Black) used under one lighting condition).

tion developed.

$$
\begin{aligned}
& {[Y]=[X][h]} \\
& {[h]=\left[X^{T} X\right]^{-1}[X]^{T}[Y]}
\end{aligned}
$$

The coefficients thus derived by solving for [h] as shown in Equation 1, provided the algorithm for transforming a colour component R, G, or B. The same operation was repeated for the 3 colour components. The set of 3 functions developed were then used to transform the colour values of the other colour squares that represent different objects in the same image in order to obtain a colour adjusted image. The final RGB values for each colour region were compared to estimate the variance among images obtained under different lighting situations. As a final measure to fine tune the algorithm, the gray level corresponding to the Gray card region (128) was adjusted to bring the total variance computed among RGB values of the new images to a minimum.

\section{RGB correction on colour palette regions}

The image of the colour palette with 3 standard colour cards and 6 refernce colours are shown in Fig. 1. The derived equations were used to correct the RGB values of the 6 colour regions in the colour palette. A sample set of such transformation functions derived for one lighting setting is given in Eqn 2-4.

$$
\begin{aligned}
& R_{\text {New }}=-0.003108 R_{\text {old }}^{2}+2.1102 R_{\text {old }}-46.8914 \\
& G_{\text {New }}=-0.002983{G_{\text {Old }}}^{2}+2.0802 G_{\text {old }}-46.2674 \\
& B_{\text {New }}=-0.003705 B_{\text {old }}^{2}+2.2362 B_{\text {old }}-48.2317
\end{aligned}
$$

Where $\mathrm{R}, \mathrm{G}, \mathrm{B}_{\mathrm{Old}}$ are the RGB values in the original image and $R, G, B_{\text {New }}$ are the RGB values of the corrected image.

In order to simulate and represent the field situations, some of the pictures were taken under natural light outside as well as in the shade. The RGB values pertaining to 10 different points in the White, Gray, and Black regions were sampled and recorded together with those sampled from the other 6 colour regions. The variance values computed for each of the colour components $R$, $\mathrm{G}$, and $\mathrm{B}$, of selected regions representing each colour palette before and after the colour adjustment are given in this Table 1. The high variance values before adjusting the colours indicate that a colour is represented by significantly different RGB values depending on the light-

Table 2. Variances observed in the RGB values among the images of a plant leaf acquired under 5 different lighting conditions before and after correction.

\begin{tabular}{lllllll|} 
& \multicolumn{2}{c}{ Red } & \multicolumn{2}{c}{ Green } & \multicolumn{2}{c|}{ Blue } \\
Card colour & Before & After & Before & After & Before & After \\
\hline Region 1 & 189 & 161 & 210 & 38 & 291 & 62 \\
Region 2 & 226 & 4 & 247 & 159 & 0 & 0 \\
Region 3 & 11 & 7 & 446 & 200 & 247 & 166 \\
Region 4 & 533 & 79 & 402 & 83 & 42 & 94 \\
Region 5 & 97 & 0 & 354 & 228 & 183 & 35 \\
Region 6 & 246 & 129 & 105 & 59 & 315 & 164 \\
Mean & 217 & 63 & 294 & 128 & 180 & 87
\end{tabular}


ing source under which it is imaged. After adjustment of colour values, the mean variances show a significant reduction. The only exception being with the RGB values pertaining to green colour imaged under fluorescent lighting. This could be attributed to the unbalanced nature of spectral distribution in fluorescent light, which may significantly alter the $\mathrm{R}$ and $\mathrm{B}$ components compared to the $\mathrm{G}$ component.

\section{RGB correction on plant leaf regions}

The colours extracted from the plant leaf were transformed using the new algorithms derived from the RGB values sampled from the standard colour cards included in the same image. The analyses showed that the algorithms derived in following the developed approach were capable of transforming the image RGB values to new values giving lower variance among the same colours in different images. The variance values computed for each of the colour components R, G, and B, of selected regions of different colour patches on the plant leaf before and after the colour adjustment procedure are shown in Table 2. Pixel value averaging was used in both these representations to minimize any variations within the selected representative region, in each lighting situation. The variance among the adjusted colour regions appear to be lower than that before adjustment, indicating the success of the method tested herein.

The algorithm as well as the procedure to acquire images was expected to be relatively easy to implement on general purpose software platforms and in-situ image acquisition work, and present more flexibility in deciding on the gray level that corresponds to the level of gray used in the image compared to other commercially available image editing software, and reduce the inter-image variability in the final colours obtained after the transformation.

\section{Flexibility of the algorithm}

There were several advantages in the new approach compared to the use of traditional colour balancing methods for RGB values of acquired images. The developed algorithm corrected RGB values by taking into account 10 representative regions distributed over the whole image representing the three colour pallets in the image. This undoubtedly makes the algorithm more robust and representative of the existing RGB values, since a close examination of the standard colour regions would reveal that there are variations in RGB values among the pixels regardless of the effort to provide uniform lighting at the time of taking the image.

Further, an additional 'tuning' operation was also built into the new algorithm whereby the RGB value of the Gray colour card could be adjusted in a manner that would provide minimum variance among the same colour regions pictured under different lighting situations, after subjecting them to the colour transformations.

\section{Conclusion}

The developed algorithm for colour adjustment of images provided less dispersed RGB values for a certain colour pictured under different lighting situations, compared to the original image. This made it possible to use images acquired in the field with minimal colour variations for subsequent image analyses and feature recognition steps. It was however necessary to acquire the image all the time with the three standard colour cards in the frame of the image together with the subject, to carry out this colour adjustment procedure.

\section{Acknowledgements}

We thank The Research Council (TRC) of the Sultanate of Oman for funding this study (Project No. RC/AGR/ SWAE/11/01-Development of Computer Vision Technology for Quality Assessment of Dates in Oman).

\section{References}

Brown, G. K.and Timm, E. J., 1992. Lighting for fruit and vegetable sorting. ASAE paper no. 936069, St. Joseph, MI, USA.

Chang, Y., Reid and J. F., 1996. RGB calibration for color image analysis in machine vision. IEEE Transactions on Image Processing. 5, 1414-1422.

Chikane, V.and Fuh, C., 2006. Automatic white balance for dgital still cameras. Journal of Information Science and Engineering. 22, 497-509.

Hehn, J. L. and Sokhansanj, S. 1990. Canola and mustard seed identification using mackintosh based imaging system. ASAE paper no. 903534, St. Joseph, MI, USA.

Li, W., Thompson, M. S., Xiong, Y. and Lange, H. 2006. A new image calibration technique for colposcopic images. Medical Imaging 2006: Image Processing, edited by Joseph M. Reinhardt, Josien P. W. Pluim, in Proceedings of SPIE. 6144, 227-239.

Liu, Z., Cheng, F., Ying, Y. and Rao, X. 2005. Identification of rice seed varieties using neutral network. Journal of Zhejiang University Science. 6B, 10951100 .

Luo, X., Jayas, D. S., Crowe, T. G. and Bulley, N. R. 1997. Evaluation of light sources for machine vision. Canadian Agricultural Engineering. 39, 309-315.

Majumdar, S. and Jayas, D.S. 1999. Classification of bulk samples of cereal grains using machine vision. Journal of Agricultural Engineering Research. 73, 35-47.

Manickavasagan, A., Sathya, G. and Jayas, D.S. 2008. Comparison of illuminations to identify wheat classes using monochrome images. Computers and Electronics in Agriculture. 63, 237-244.

Murakami, P. F., Turner, M.R., Van den Berg, A. K. and Schaberg, P. G. 2005. An instructional guide for leaf color analysis using digital imaging software. Gen. 
Tech. Rep. NE-327. Newtown Square, PA: U.S. Department of Agriculture, Forest Service, Northeastern Research Station. 33 p.

O’Neal, M., Landis, D. A.and Isaacs, R. 2002. An inexpensive, accurate method for measuring leaf area and defoliation through digital image analysis. Journal of Economic Entomology. 95, 1190 - 1194.

Paliwal, J., Visen, N. S., Jayas, D. S. and White, N.D.G. 2003. Cereal grain and dockage identification using machine vision. Biosystems Engineering. 85, 51-57.

Shahin, M. A. and Symons, S. J. 2003. Color calibration of scanners for scanner-independent grain grading. Cereal Chemistry Journal. 80, 285-289.

Takemura, Y, and Ishii, K. 2011. Auto color calibration algorithm using neural networks and its application to RoboCup robot vision. International Journal of Artificial Intelligence.

Weng, C., Chen, H. and Fuh, C. 2006. A novel automatic white balance method For digital still cameras. Journal of Communication Engineering. 12. 